\title{
Application of Dressing Method for Long Wave-Short Wave Resonance Interaction Equation
}

\author{
Maria Yurove* \\ Institute of Nuclear Physics \\ Moscow State University \\ Vorobjovy Gory \\ 119899 Moscow \\ Russia
}

\begin{abstract}
In this paper we investigate the application of Zakharov - Shabat dressing method to $(2+1)$ dimensional long wave - short wave resonance interaction equation (LSRI). Using this method we can construct the exact $N$ - soliton solution of this equation depending on arbitrary constants. It contains both solutions which don't decay along $N$ different directions in space infinity, and "dromion" ones, or localized solutions that decay exponentially in all directions.
\end{abstract}

\section{INTRODUCTION}

We investigate the $(2+1)$ - dimensional equation describing the interaction of a long wave with a packet of short waves. Such process can arise in fluid mechanics. If $L$ is a long interfacial wave and $S$ is a short waves packet, their interaction on $(x, y)$-plane is described by the system [2]

$$
\begin{aligned}
i \partial_{t} S & +i c_{g} \partial_{x} S-\beta L S-\gamma \partial_{x x}^{2} S-\delta|S|^{2} S=0, \\
\partial_{t} L & +c_{l} \partial_{y} L+\alpha \partial_{x}|S|^{2}=0,
\end{aligned}
$$

where $c_{l}$ is a long wave phase velocity along the $y$-axis, $c_{g}$ is a group velocity of a packet of short waves along the $x$-axis, and $\alpha, \beta, \gamma, \delta$ are constant parameters of a system under consideration. Using the "resonance condition" $c_{l}=c_{g}[3]$, some linear coordinate transformations and scale transformation of the constants, it is possible to rewrite this system in the form:

$$
\begin{aligned}
i \partial_{t} S & -\partial_{x x}^{2} S+L S=0, \\
\partial_{y} L & =2 \partial_{x}|S|^{2} .
\end{aligned}
$$

The integrability of this system has been established earlier. Therefore, a Lax pare for equation (1)has been constructed in [4], and an exact solution for this equation has been presented in [2]. The LSRI equation considered in [5] and [6] can be written down in our form by simple linear coordinate transformations. In 5 the authors have performed a deep analysis of different classes of exact solutions such as "positon", "dromion" and "solitoff", and they have derived a number of new solutions including one on a continuous wave background. The recent investigation of the above system has been performed in [6]. The authors showed that (1) possess the Painleve property and they generated an extended class of periodic wave solutions and exponentially localized ones.

In this paper we consider the application of Zakharov - Shabat dressing method [1] to the above system. Using the general scheme we obtain the exact $N$ - soliton solution which depends on arbitrary constants. The general form of this solution is new. The analysis of asymptotic behaviour demonstrates that this solution describes an interaction of $N$ solitons and does not decay along $N$ different directions in space infinity. For $N=1$ the asymptotic form of the solution obtained coincides with the solution of [2]. If we impose some restrictions on the constants we obtain the "dromion" i.e. localized solutions, that decay exponentially in all directions.

\section{THE DRESSING METHOD}

Now, let us reformulate the system under consideration in the coordinates $\xi$ and $\eta$, where

$$
\partial_{\xi}=\partial_{x}-\partial_{y}, \partial_{\eta}=\partial_{x}
$$

*Electronic address: yurova-m@rambler.ru 
to apply the general inverse scattering problem method for integration of nonlinear differential equations, proposed by Zakharov and Shabat [1]. Then equation (1) has the form

$$
\begin{array}{r}
i \partial_{t} S-\partial_{\eta \eta}^{2} S+L S=0 \\
\left(\partial_{\eta}-\partial_{\xi}\right) L=2 \partial_{\eta}|S|^{2} .
\end{array}
$$

Following the above method [1], we consider a linear integral operator $\widehat{F}$, acting to function $\phi$

$$
\widehat{F} \phi(\xi, \eta, t)=\int_{-\infty}^{\infty} F(\xi, z, \eta, t) \phi(z, \eta, t) d z
$$

where the matrix $F$ satisfy the condition

$$
\sup _{\xi>\xi_{0}} \int_{\xi_{0}}^{\infty}|F(\xi, z, \eta, t)| d z<\infty
$$

for all $\xi_{0}>-\infty$. Let the operator $\widehat{F}$ have the form

$$
\widehat{1}+\widehat{F}=\left(\widehat{1}+\widehat{K}_{+}\right)^{-1}\left(\widehat{1}+\widehat{K}_{-}\right),
$$

where $\widehat{K}_{+}$and $\widehat{K}_{-}$are Volterra operators

$$
\pm \widehat{K}_{ \pm} \phi(\xi, \eta, t)=\int_{\xi}^{ \pm \infty} K_{ \pm}(\xi, z, \eta, t) \phi(z, \eta, t) d z,
$$

and $K_{+}(\xi, z, \eta, t)=0$ for $z<\xi, K_{-}(\xi, z, \eta, t)=0$ for $z>\xi$. The kernel $K_{+}$satisfy the Gelfand-Levitan-Marchenko equation

$$
F(\xi, z)+K_{+}(\xi, z)+\int_{\xi}^{\infty} K_{+}(\xi, s) F(s, z) d s=0(z>\xi)
$$

Now let us define two primeval operators, acting to function $\phi$ in the form:

$$
\begin{aligned}
& \widehat{L_{0}^{(1)}}=\partial_{t}-i \sigma^{+} \partial_{\xi \xi}^{2}, \\
& \widehat{L_{0}^{(2)}}=\partial_{\eta}-\sigma^{+} \partial_{\xi}, \sigma^{+}=\left(\begin{array}{ll}
1 & 0 \\
0 & 0
\end{array}\right),
\end{aligned}
$$

and consider a class of differential operators $\widehat{L^{(1)}}, \widehat{L^{(2)}}$, obtained from $\widehat{L_{0}^{(1)}}$ and $\widehat{L_{0}^{(2)}}$ by the transformation:

$$
\widehat{L^{(i)}}\left(\widehat{1}+\widehat{K_{+}}\right)=\left(\widehat{1}+\widehat{K_{+}}\right) \widehat{L_{0}^{(i)}}, i=1,2
$$

where $\widehat{1}+\widehat{K_{+}}$is Volterra operator. Since the differential parts of operator relation (10) are equal to zero, $\widehat{L^{(1)}}$ and $\widehat{L^{(2)}}$ have the form:

$$
\widehat{L^{(1)}}=\widehat{L_{0}^{(1)}}+u_{1}^{(1)} \partial_{\xi}+u_{0}^{(1)}, \widehat{L^{(2)}}=\widehat{L_{0}^{(2)}}+u_{0}^{(2)} .
$$

Equation (10) gives the reccurent relations between the matrix coefficients $u_{k}^{(i)}, i=1,2$, and components of matrix kernel $K_{+}(\xi, z, \eta, t)$. It turned out, that if the components of $K_{+}$satisfy some conditions, we can obtain the Lax pare for the system (1). Let

$$
K_{+}(\xi, z, \eta, t)=\left(\begin{array}{ll}
k_{11} & k_{12} \\
k_{21} & k_{22}
\end{array}\right), k_{i j}=k_{i j}(\xi, z, \eta, t)
$$

Then, if we impose the following conditions:

$$
\begin{array}{r}
\left(\partial_{\eta}-\partial_{\xi}-k_{22}\right)\left(\left.k_{12}\right|_{z=\xi}\right)+\left.\partial_{z} k_{12}\right|_{z=\xi}=0 \\
\left(\partial_{\eta}+k_{11}\right)\left(\left.k_{21}\right|_{z=\xi}\right)-\left.\partial_{z} k_{21}\right|_{z=\xi}=0 \\
\left.k_{12}\right|_{z=\xi}=-\left.\bar{k}_{21}\right|_{z=\xi},\left.\quad k_{11}\right|_{z=\xi}=\left.\bar{k}_{11}\right|_{z=\xi}
\end{array}
$$


we can identify a short waves packet $S$ and a long interfacial wave $L$ with $k_{i j}$ as follow:

$$
\begin{aligned}
S & =\left.k_{21}(\xi, z, \eta, t)\right|_{z=\xi} \\
L & =2\left[S \bar{S}-\partial_{\xi}\left(\left.k_{11}(\xi, z, \eta, t)\right|_{z=\xi}\right)\right] .
\end{aligned}
$$

Thus, the matrix coefficients $u_{k}^{(i)}$ can be expressed as the functions of $S$ and $L$ :

$$
\begin{aligned}
u_{0}^{(1)} & =i\left(\begin{array}{cc}
-S \bar{S}+L & \left(\partial_{\eta}+\partial_{\xi}\right) \bar{S} \\
-\partial_{\eta} S & -S \bar{S}
\end{array}\right), \\
-i u_{1}^{(1)} & =u_{0}^{(2)}=\left(\begin{array}{cc}
0 & \bar{S} \\
S & 0
\end{array}\right) .
\end{aligned}
$$

Consequently, it is possible to demonstrate by straightforward calculation, that the integrability condition for the "dressing" operators (11) leads to equation (1) under consideration, and we obtain two commuting operators

$$
\left[\widehat{L^{(1)}}, \widehat{L^{(2)}}\right]=0
$$

that constitute the Lax pare for equation (1). The same form of Lax pare has been obtained in [4].

\section{EXACT SOLUTION}

Now, let us construct the exact solution of LSRI equation using the above method. Two commutation relations for operator $\widehat{F}$

$$
\left[\widehat{L_{0}^{(1)}}, \widehat{F}\right]=0, \quad\left[\widehat{L_{0}^{(2)}}, \widehat{F}\right]=0
$$

give two differential equations for the kernel $F$ :

$$
\begin{aligned}
& \partial_{t} F-i \sigma^{+} \partial_{\xi}^{2} F-i \partial_{z}^{2} F \sigma^{+}=0, \\
& \partial_{\eta} F-\sigma^{+} \partial_{\xi} F-\partial_{z} F \sigma^{+}=0 .
\end{aligned}
$$

We choose the solution of these equations in the form:

$$
\begin{aligned}
F & =\sum_{n}^{N}\left(\begin{array}{cc}
f_{11}^{n} & f_{12}^{n} \\
f_{21}^{n} & f_{22}^{n}
\end{array}\right), \\
f_{11}^{n} & =\left(f_{11}^{n}\right)_{0} e^{-\kappa_{1}^{n}(2 \eta+\xi+z)}, \\
f_{12}^{n} & =\left(f_{12}^{n}\right)_{0} e^{i\left(\kappa_{2}^{n}\right)^{2} t-\kappa_{2}^{n}(\eta+\xi)-\bar{\kappa}_{3}^{n} z}, \\
f_{21}^{n} & =-\left(\bar{f}_{12}^{n}\right)_{0} e^{-i\left(\bar{\kappa}_{2}^{n}\right)^{2} t-\bar{\kappa}_{2}^{n}(\eta+z)-\kappa_{3}^{n} \xi}, \\
f_{22}^{n} & =\left(f_{22}^{n}\right)_{0} e^{-\kappa_{4}^{n}(\xi+z)},
\end{aligned}
$$

where

$$
\Re\left(f_{i j}^{n}\right)_{0}>0, i, j=1,2, \Im\left(f_{11}^{n}\right)_{0}=\Im\left(f_{22}^{n}\right)_{0}=0,
$$

and $\kappa_{i}^{n}=\bar{\kappa}_{i}^{n}, i=1,4, \kappa_{i}^{n}=\Re \kappa_{i}^{n}+i \Im \kappa_{i}^{n}, i=2,3, \Re \kappa_{i}>0, i=1, . .4$ are constant parameters. Solving the equation (8), we obtain the explicit form of the matrix kernel $K_{+}(\xi, z, \eta, t)$, which is too complicated and we omit it here. Further using the expressions (14) we can write down the exact $N$ - solitons solution for the short waves packet $S$ and a long interfacial wave $L$. Returning to usual space - time variables $x, y, t$, we obtain the explicit form of our solution:

$$
\begin{aligned}
S & =\sum_{n}^{N} \frac{1}{\operatorname{det} A}\left|\begin{array}{cc}
A & B^{n} \\
\alpha & \beta^{n}
\end{array}\right| \\
L & =2\left[S \bar{S}-\left(\partial_{x}-\partial_{y}\right) \sum_{n}^{N} \frac{1}{\operatorname{det} A}\left|\begin{array}{cc}
A & B^{n} \\
\tilde{\alpha} & \tilde{\beta^{n}}
\end{array}\right|\right]
\end{aligned}
$$


where the block $4 N \times 4 N$ matrix $A$, the $4 N \times 1$ column $B^{n}$, the $1 \times 4 N$ rows $\alpha$ and $\tilde{\alpha}$ and the scalars $\beta^{n}$ and $\tilde{\beta^{n}}$ have the form:

$$
\begin{aligned}
& A=\left(\begin{array}{cccc}
I_{N}+a_{11} & a_{12} & 0 & 0 \\
a_{21} & a_{22} & I_{N} & 0 \\
0 & I_{N} & a_{33} & a_{34} \\
0 & 0 & a_{43} & I_{N}+a_{44}
\end{array}\right) \\
& B^{n}=\left(\begin{array}{c}
e^{\kappa_{1}^{n} y}\left(a_{11}\right)_{i n} \\
e^{\kappa_{1}^{n} y}\left(a_{21}\right)_{i n} \\
e^{\bar{\kappa}_{2}^{n} y}\left(a_{33}\right)_{i n} \\
e^{\bar{\kappa}_{2}^{n} y}\left(a_{43}\right)_{i n}
\end{array}\right), \\
& \alpha=\left(\begin{array}{lll}
0 & 0 & e^{-\bar{\kappa}_{2}^{n} x-\left(\bar{\kappa}_{2}^{n}-\kappa_{3}^{n}\right) y}\left(\bar{f}_{12}^{n}\right)_{0} e^{-i\left(\bar{\kappa}_{2}^{n}\right)^{2} t} e^{\kappa_{4}^{n} y}\left(f_{22}^{n}\right)_{0}
\end{array}\right), \\
& \tilde{\alpha}=\left(\begin{array}{llll}
e^{-2 \kappa_{1}^{n} x-\kappa_{1}^{n} y}\left(f_{11}^{n}\right)_{0}-e^{-\kappa_{2}^{n} x}\left(f_{12}^{n}\right)_{0} e^{i\left(\kappa_{2}^{n}\right)^{2} t} & 0 & 0
\end{array}\right), \\
& \beta^{n}=e^{\bar{\kappa}_{2}^{n} y} \alpha_{n}, \tilde{\beta^{n}}=e^{\kappa_{1}^{n} y} \tilde{\alpha}_{n} ;
\end{aligned}
$$

$I_{N}$ is $N \times N$ unit matrix and 0 are $N \times N$ or $1 \times N$ matrices respectively, $a_{I J} \equiv\left\|\left(a_{I J}\right)_{i j}\right\|$ are $N \times N$ matrices with components:

$$
\begin{aligned}
\left(a_{11}\right)_{i j} & =\frac{e^{\left(\kappa_{1}^{i}-\kappa_{1}^{j}\right) y-2 \kappa_{1}^{j} x}\left(f_{11}^{j}\right)_{0}}{\kappa_{1}^{i}+\kappa_{1}^{j}}, \\
\left(a_{21}\right)_{i j} & =\frac{e^{\left(\bar{\kappa}_{2}^{i}-\kappa_{1}^{j}\right) y-2 \kappa_{1}^{j} x}\left(f_{11}^{j}\right)_{0}}{\bar{\kappa}_{2}^{i}+\kappa_{1}^{j}}, \\
\left(a_{12}\right)_{i j} & =\frac{-e^{\kappa_{1}^{i} y-\kappa_{2}^{j} x}\left(f_{12}^{j}\right)_{0}}{\kappa_{1}^{i}+\kappa_{2}^{j}} e^{i\left(\kappa_{2}^{j}\right)^{2} t}, \\
\left(a_{22}\right)_{i j} & =\frac{-e^{\bar{\kappa}_{2}^{i} y-\kappa_{2}^{j} x}\left(f_{12}^{j}\right)_{0}}{\bar{\kappa}_{2}^{i}+\kappa_{2}^{j}} e^{i\left(\kappa_{2}^{j}\right)^{2} t}, \\
\left(a_{33}\right)_{i j} & =\frac{e^{\left(\bar{\kappa}_{3}^{i}+\kappa_{3}^{j}-\bar{\kappa}_{2}^{j}\right) y-\bar{\kappa}_{2}^{j} x}\left(\bar{f}_{12}^{j}\right)_{0}}{\bar{\kappa}_{3}^{i}+\kappa_{3}^{j}} e^{-i\left(\bar{\kappa}_{2}^{j}\right)^{2} t}, \\
\left(a_{43}\right)_{i j} & =\frac{e^{\left(\kappa_{4}^{i}+\kappa_{3}^{j}-\bar{\kappa}_{2}^{j}\right) y-\bar{\kappa}_{2}^{j} x}\left(\bar{f}_{12}^{j}\right)_{0}}{\kappa_{4}^{i}+\kappa_{3}^{j}} e^{-i\left(\bar{\kappa}_{2}^{j}\right)^{2} t}, \\
\left(a_{34}\right)_{i j} & =\frac{-e^{\left(\bar{\kappa}_{3}^{i}+\kappa_{4}^{j}\right) y}\left(f_{22}^{j}\right)_{0}}{\bar{\kappa}_{3}^{i}+\kappa_{4}^{j}},\left(a_{44}\right)_{i j}=\frac{e^{\left(\kappa_{4}^{i}+\kappa_{4}^{j}\right) y}\left(f_{22}^{j}\right)_{0}}{\kappa_{4}^{i}+\kappa_{4}^{j}} .
\end{aligned}
$$

In general case this solution describes the intersection of $N$ solitons. The analysis of its asymptotic behaviour demonstrates that it vanishes at spatial infinity $x, y \rightarrow \infty$ in all directions except the $N$ ones

$$
\frac{y}{x}=\frac{\Re \kappa_{2}^{i}}{\Re \kappa_{3}^{i}}, i=1, \ldots N .
$$

In the simplest case of $N=1$ the one - soliton solution for the short waves packet $S$ has the form:

$$
S=\frac{g(x, y, t)}{f(x, y, t)}
$$

where

$$
\begin{aligned}
g & =\left(\bar{f}_{12}\right)_{0} e^{-i\left(\bar{\kappa}_{2}\right)^{2} t-\kappa_{3} y+\bar{\kappa}_{2} x}\left[1-\frac{e^{-2 \kappa_{1} x}\left(f_{11}\right)_{0}\left(\kappa_{1}-\bar{\kappa}_{2}\right)}{2 \kappa_{1}\left(\kappa_{1}+\bar{\kappa}_{2}\right)}\right] \times \\
& \times\left[1-\frac{e^{2 \kappa_{4} y}\left(f_{22}\right)_{0}\left(\kappa_{4}-\kappa_{3}\right)}{2 \kappa_{4}\left(\kappa_{4}+\kappa_{3}\right)}\right],
\end{aligned}
$$




$$
\begin{aligned}
f & =\frac{\left|\left(f_{12}\right)_{0}\right|^{2} e^{2 \Re \kappa_{3} y-2 \Re \kappa_{2} x-4 \Re \kappa_{2} \Im \kappa_{2} t}}{4 \Re \kappa_{2} \Re \kappa_{3}} \times \\
& \times\left[1+\frac{e^{-2 \kappa_{1} x}\left(f_{11}\right)_{0}\left|\kappa_{1}-\kappa_{2}\right|^{2}}{2 \kappa_{1}\left|\kappa_{1}+\kappa_{2}\right|^{2}}\right]\left[1+\frac{e^{2 \kappa_{4} y}\left(f_{22}\right)_{0}\left|\kappa_{4}-\kappa_{3}\right|^{2}}{2 \kappa_{4}\left|\kappa_{4}+\kappa_{3}\right|^{2}}\right]+ \\
& +\left[1+\frac{e^{-2 \kappa_{1} x}\left(f_{11}\right)_{0}}{2 \kappa_{1}}\right]\left[1+\frac{e^{2 \kappa_{4} y}\left(f_{22}\right)_{0}}{2 \kappa_{4}}\right]
\end{aligned}
$$

and one may see that its asymptotic form at $x, y \rightarrow \infty$ is

$$
S \sim \frac{e^{-i \Re \varphi(x, y, t)}}{\cosh \left(\Re \kappa_{3} y-\Re \kappa_{2} x-2 \Re \kappa_{2} \Im \kappa_{2} t+\ln \frac{|c|}{\sqrt{\Re \kappa_{2} \Re \kappa_{3}}}\right)}
$$

that coincides with the solution obtained in [2].

As it has been established earlier, the equations under consideration admit a "dromion" solution, i.e. a localized solution that decays exponentially in all directions. In our case if we impose the conditions

$$
\kappa_{1}=\Re \kappa_{2}, \Im \kappa_{2}=0, \kappa_{4}=\Re \kappa_{3}, \Im \kappa_{3}=0,
$$

we obtain the exponentially localized $N$ - dromion solution for $S$. It looks like (20), but the block matrices $A, B^{n}, \alpha$ and $\tilde{\alpha}$ are $2 N \times 2 N, 2 N \times 1$ and $1 \times 2 N$ respectively. The $N=1$ - dromion solution has the form :

$$
S=\frac{\left(f_{12}\right)_{0} e^{-i\left(\kappa_{1}\right)^{2} t} \operatorname{sech}\left(\kappa_{1} x+c_{1}\right) \operatorname{sech}\left(\kappa_{4} y+c_{4}\right)}{4\left[\zeta+\tanh \left(\kappa_{1} x+c_{1}\right)+\tanh \left(\kappa_{4} y+c_{4}\right)\right]},
$$

where the constants are:

$$
\begin{array}{r}
\zeta^{2}=\frac{\left|\left(f_{12}\right)_{0}\right|^{2}+\left(f_{11}\right)_{0}\left(f_{22}\right)_{0}}{4 \kappa_{1} \kappa_{4}} \\
e^{2 c_{1}}=\frac{\left(f_{22}\right)_{0}}{2 \kappa_{4}(\zeta+2) \zeta}, e^{2 c_{4}}=e^{2 c_{1}} \zeta^{2} .
\end{array}
$$

This solution close to one constructed in [6].

\section{CONCLUSION}

Using the Zakharov - Shabat dressing method we constructed $N$ - soliton solution for $(2+1)$ - dimensional long wave - short wave resonance interaction equation (LSRI). We demonstrated that in generic situation this solution does not decay in $N$ preferred directions in space infinity. Some choice of constants leads to a localized solutions that decay exponentially in all directions.

The finding of our study may elicit further investigation. It would be interesting to analyze in more depth both the solution obtained for the different choices of constants, and another solutions, as well as their properties end time evolution.

[1] V.E.Zakharov, A.B.Shabat, Funct. Anal. Appl. 8 226, 1974.

[2] V.K.Melnikov, JINR-P2-84-13, Jan 1984.

[3] R.K.Dodd, J.C.Eilbeck, J.D.Gibbon, H.C.Morris, "Solitons and Nonlinear Wave Equations", Academic Press Inc.(London) Ltd., 1984, and references therein.

[4] S.P.Novikov, S.V.Manakov, L.P.Pitaevskii, V.E.Zakharov, Theory of Solitons. The Method of Inverse Scattering (New York:Plenum), 1984.

[5] Derek W.C.Lai, Kwok W.Chow, Journal of Physical Society of Japan, 68, 6 1847, 1999.

[6] R.Radha, C.S.Kumar, M.Lakshmanan, X.Y.Tang, S.Y.Lou, Journal of Physics A: Mathematical and General, 38, 44 9649, 2005. 